\title{
A simple strategy to design broadband low power consumption distributed Raman amplifier
}

\author{
Renan S. Santos, Maria Aparecida G. Martinez, \\ Centro Federal de Educação Tecnológica Celso Suckow da Fonseca, Photonics Lab, Electrical \\ Engineering Department, Avenida Maracanã, 229, Rio de Janeiro, Brazil, 20271-110, \\ renansilvasantos@gmail.com, magmartinez@gmail.com \\ Maria Thereza M. R. Giraldi \\ Instituto Militar de Engenharia, Photonics Lab,Electrical Engineering Department, Praça General Tibúrcio, \\ 80, Rio de Janeiro, Brazil, 22290-270, mtmrocco@ime.eb.br
}

\begin{abstract}
In this work, a simple strategy to design wideband low power consumption Raman amplifiers is demonstrated for a threepump configuration using a low water peak optical fiber. The approach is based on the introduction of a novel numerical measure, which quantifies and isolates pump-pump interaction contribution to gain profile and analyzes its correlation to amplifier minimum ripple. The method tailors the amplifier gain spectrum over $80 \mathrm{~nm}$ bandwidth with a ripple smaller than $1 \mathrm{~dB}$, a gain on the order of $4 \mathrm{~dB}$ for up to $75 \mathrm{~km}$ fiber length, and a total pump power consumption smaller than $300 \mathrm{~mW}$.
\end{abstract}

Index Terms - Optical Communications, Raman Amplifiers, Raman Amplifier Optimization

\section{INTRODUCTION}

Recent advances in Internet-based applications accessible for both commercial and residential customers as well as evolving social networks still increases the traffic growth throughout the network. Additionally, low price mobile services, are also forcing a huge increment on the capacity demand [1]. Among the key technologies to respond to these needs are low loss modern fibers, which make possible 40THz optical window (the O, E, S, C and L bands) system operation, and wideband Raman optical amplifiers.

Over the past years, techniques and methodologies to design wideband Raman amplifiers have been reported in the literature [2-8]. These techniques use multiple pumps, around five to eight, to achieve an $80 \mathrm{~nm}$ bandwidth with a ripple better than $0.1 \mathrm{~dB}$. The drawback of such approaches is the high power consumption in the Raman amplification module, and the use of complex and time consuming numerical optimization routines to find the best combination of pump power and wavelengths [9-10]. Table I shows previous works reported in the literature regarding the number of pumps lasers, total power consumption and ripple for Raman amplifiers with approximately $80 \mathrm{~nm}$ bandwidth. 
Table I: RAMAN AMPLIFIER PUMP SETUPS REPORTED IN LITERATURE

\begin{tabular}{cccc}
\hline Reference & $\begin{array}{c}\text { Number of pump } \\
\text { lasers }\end{array}$ & $\begin{array}{c}\text { Total pump power } \\
(\mathbf{m W})\end{array}$ & Ripple (dB) \\
\hline Añia-Castanon, 2007 [2] & 3 & 1000 & $<1.80$ \\
Lee, 2003 [5] & 5 & 900 & $<1.00$ \\
Liu, 2004 [6] & 5 & 800 & $<0.76$ \\
Perlin, 2002 [7] & 16 & 530 & $<0.05$ \\
\hline
\end{tabular}

In addition, since the interest concerning environmental issues is intensifying, the use of low power consumption devices and systems will be a necessity in the near future. Consequently, future networks should have the capacity to provide a massive quantity of bandwidth in an energy efficient way [1]. Another concern in modern long-haul broadband fiber optic communication systems using wavelength division multiplexing (WDM) is the minimization of the amplifier ripple [11-12]. In this work, we introduce an approach to design wideband low power consumption Raman amplifier over an $80 \mathrm{~nm}$ band. It lies on isolating the pump-pump interaction contribution to wideband Raman amplifiers spectrum and studying its relation to the amplifier ripple, through the introduction of a novel design strategy. In section II, the approach along with the scenario of interest are introduced, accompanied by a discussion on minimum total power conditions for pump-pump interaction to affect gain profile. In section III, the results and analysis are conducted for counter and co-propagating configuration for fiber lengths up to $75 \mathrm{~km}$; up to $80 \mathrm{~nm}$ bandwidth and total pump power smaller than $300 \mathrm{~mW}$. Finally, the conclusions are reported in section IV.

\section{DESCRIPTION OF THE APPROACH}

The scenario of interest is shown in Fig.1. It consists of three pump lasers in co or counterpropagating configuration. The fiber span length, $\mathrm{L}$, ranges from $25 \mathrm{~km}$ up to $75 \mathrm{~km}$. The signals are within the $\mathrm{S}, \mathrm{C}$ and $\mathrm{L}$ (from 1510 to $1590 \mathrm{~nm}$ ) fiber communication bands, and at $0.1 \mathrm{~mW} /$ channel. The three equally spaced pumps wavelength are $\lambda_{1}=1420 \mathrm{~nm}, \lambda_{2}=1450 \mathrm{~nm}$ and $\lambda_{3}=1480 \mathrm{~nm}$, and its power values are denoted by $\mathrm{P}_{1}, \mathrm{P}_{2}$ and $\mathrm{P}_{3}$, respectively.

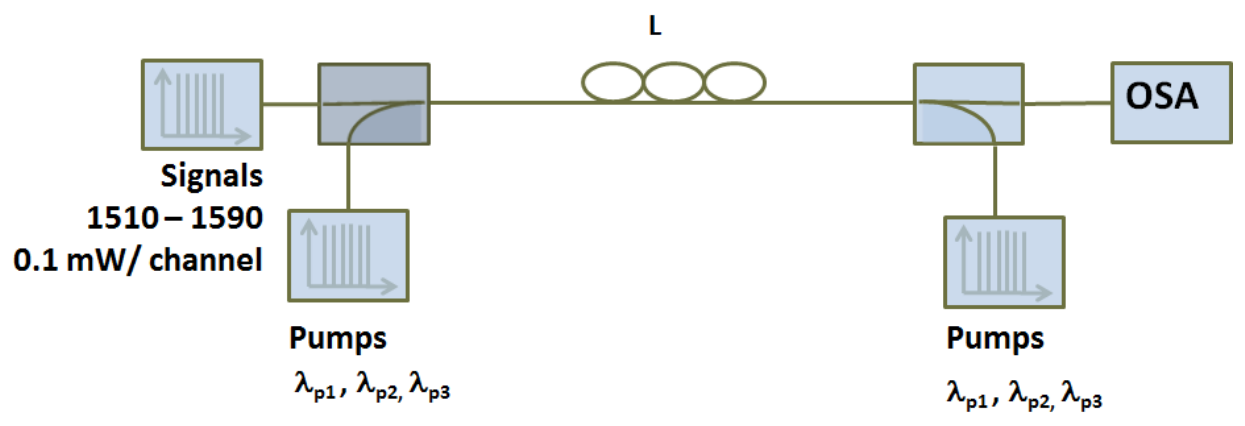

Fig. 1 Scenario of interest 
In broadband Raman amplifier the ripple is greatly affected by pump-pump interaction [13]. For the scenario described in Figure 1, pump-pump interaction dominates the amplifier ripple for equal pump power levels greater than a certain threshold value $\left(\mathrm{P}_{1}=\mathrm{P}_{2}=\mathrm{P}_{3}>\mathrm{P}_{\text {th }}\right)$. Pump-pump interaction signature is the typical spectrum tilt, with higher gain in the longer wavelength side than in the shorter wavelength side of the amplification band (roughly ranging from $1515 \mathrm{~nm}$ up to $1585 \mathrm{~nm}$ ). Therefore, the amount of pump-pump interaction contributing to gain ripple can be estimated through $\Delta G_{\text {on-off }}$, a gain difference, defined as:

$$
\Delta G_{o n-o f f}=G_{o n-o f f}-G_{o n-o f f}^{p-p o f f}
$$

In equation (1), $G_{\text {on-off }}$ is the amplifier on-off gain and $G_{o n-o f f}^{p-p o f f}$ is the amplifier on-off gain in the absence of pump-pump interaction effect. $\Delta G_{\text {on-off }}$ is calculated through the solution of the Raman coupled nonlinear equations previously reported in the literature [14-16]. The model accounts for pump-signal interaction, pump-pump interaction, signal-signal interaction, Double Rayleigh Backscattering (DRB) and Amplified Spontaneous Emission (ASE). Further, all fields in the optical fiber were considered depolarized. The equations used can be found in the Appendix, where a parameter $\varepsilon$ is introduced in the Raman coupled equations (equations 2 and 3 in the Appendix) where it can assume either 0 or 1 value. If $\varepsilon=1$ pump-pump interaction effects are accounted in the amplifier simulation performance, and if $\varepsilon=0$ otherwise.

The design approach is based on targeting $\Delta \boldsymbol{G}_{\boldsymbol{o n} \text { - } \boldsymbol{o f f}}$ to zero, controlling just one design parameter, namely, the power value of the central pump $\left(\lambda_{2}\right)$. The central pump efficiently controls pump-pump interaction mitigating its harmful effects to gain ripple. The approach is valid for $\mathrm{P}_{\mathrm{i}}>\mathrm{P}_{\text {th }}$ where $\mathrm{i}=1,3$ and $\mathrm{P}_{1}=\mathrm{P}_{3}$. Further, the correlation between the amplifier ripple and $\Delta \boldsymbol{G}_{\boldsymbol{o n} \text {-of } \boldsymbol{f}}$ is monitored with the introduction of the relative difference between the amplifier ripple and the maximum absolute value of $\Delta \boldsymbol{G}_{\boldsymbol{o n}-\boldsymbol{o f f}}$ in the amplifier band, defined as $\Delta=\left[\right.$ Ripple $\left.-\max \left|\Delta G_{\text {On-off }}\right|\right] /$ Ripple.

\section{RESULTS}

The analysis is conducted using a True Wave (TW) Reach fiber from OFS Fitel. The TW fiber Raman gain efficiency spectrum is shown in Fig. 2 and its peak is about 2 times greater than the SMF peak value. The effective area of the TW fiber at $1450 \mathrm{~nm}$ and $1550 \mathrm{~nm}$ are $49.09 \mu \mathrm{m}^{2}$ and $56.06 \mu \mathrm{m}^{2}$, respectively, a $20 \%$ reduction compared to SMF fibers. Further, the attenuation coefficient is smaller than $0.3 \mathrm{~dB} / \mathrm{km}$ around $1400 \mathrm{~nm}$ making it a good choice to wideband distributed Raman amplification. The linear and nonlinear parameters provided by the manufacturer such as, absorption coefficient, Brazilian Microwave and Optoelectronics Society-SBMO received 24 May 2017; for review 25 May 2017 ; accepted 05 Aug 2017 Brazilian Society of Electromagnetism-SBMag 
Raman spectrum, effective area, Rayleigh coefficient and non-linear index, are input parameters to the numerical solutions of the nonlinear equations.

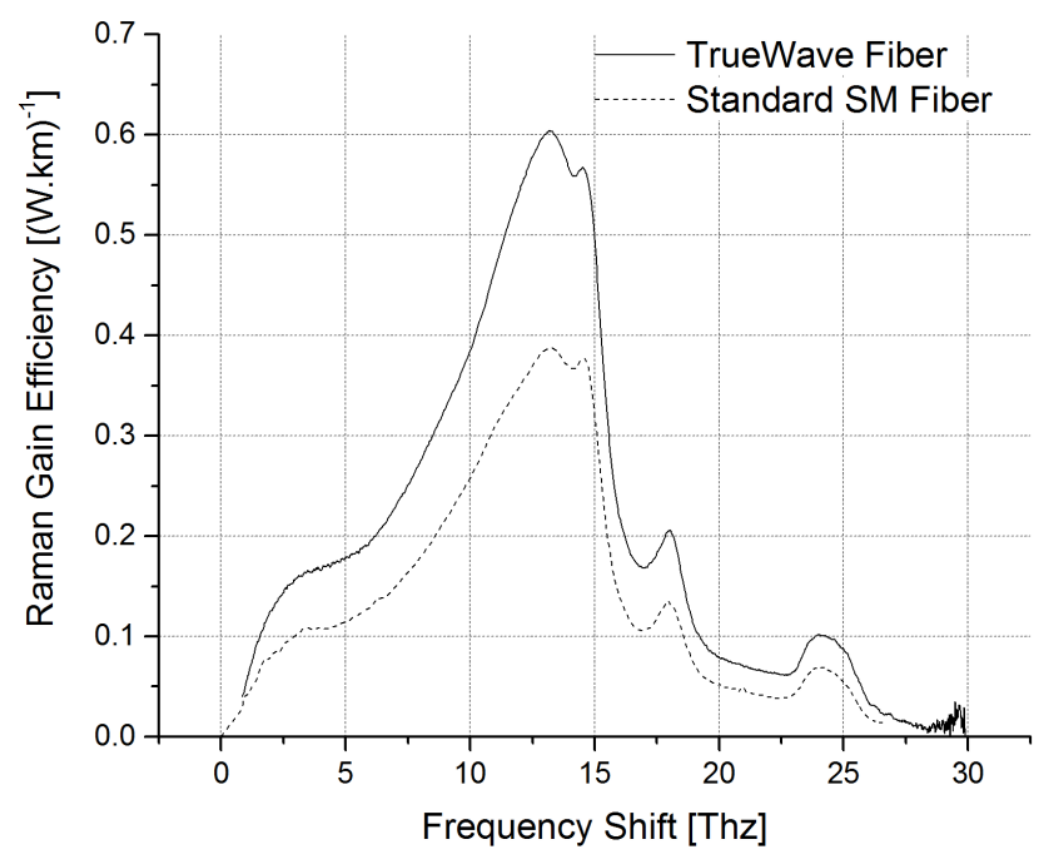

Fig.2 - TW and Standard SM fiber Raman spectrum for a pump wavelength at 1453nm

Pump-pump interaction effect in Raman amplifers leads to a power transfer from the shorter to the longer wavelength within the amplifier gain band [13]. For the scenario under consideration this signature is depicted in the set of curves in Fig. 3, where solid and dashed lines correspond to $G_{\text {on-off }}$ and $G_{\text {on-off }}^{\text {p-p off }}$, respectively. For signals below 1543nm, $G_{\text {on-off }}^{\text {p-p off }}>G_{\text {on-off }}$ indicating the amount of energy taken from the shorter wavelengths due to pump-pump interaction. The energy transferred to the longer wavelengths is noticeable above $1543 \mathrm{~nm}$ where $\mathrm{G}_{\mathrm{on}-\mathrm{off}}^{\mathrm{p}-\mathrm{p} \text { off }}<\mathrm{G}_{\text {on-off. }}$. The power transfer increases with pump power and reaches a $6 \mathrm{~dB}$ difference for the $200 \mathrm{~mW}$ set of curves. 


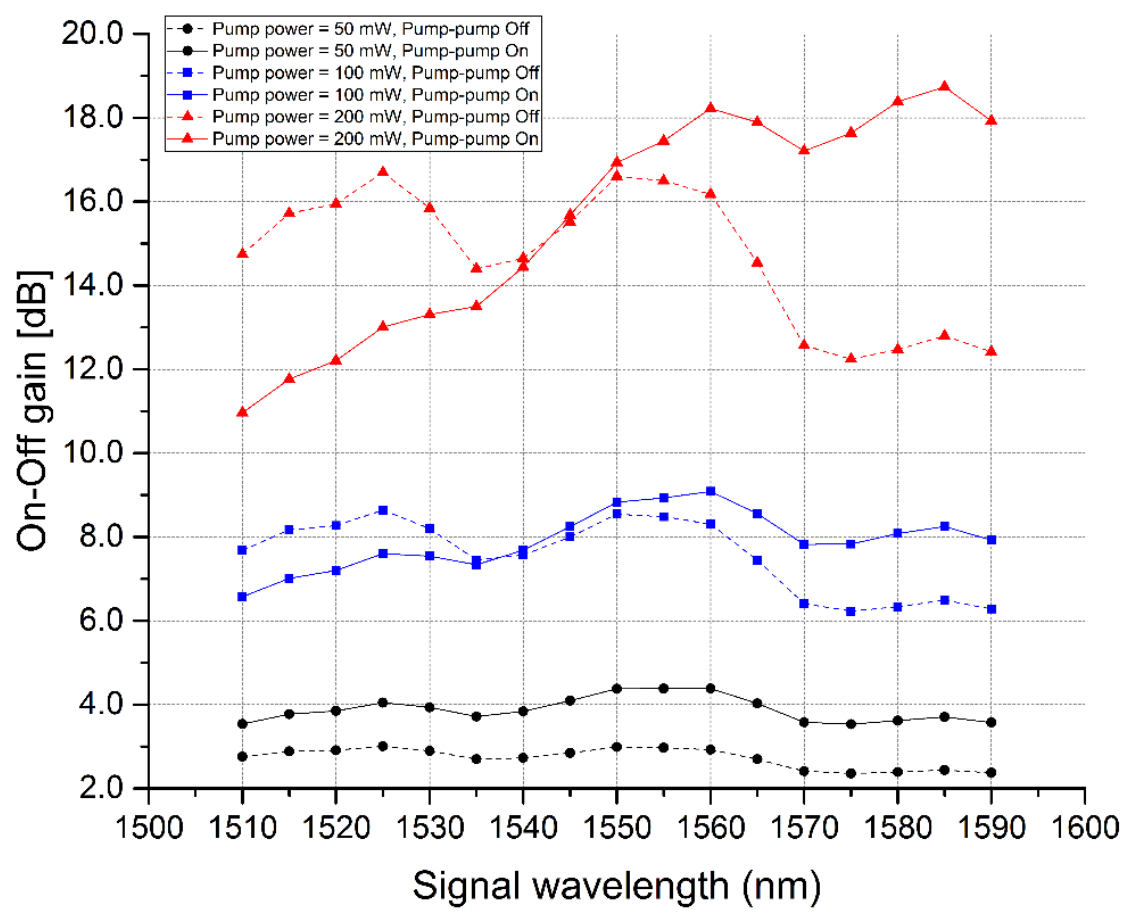

Fig.3 - On-Off gain with (solid lines) and without (dashed lines) pump-pump interaction for co-propagating pumps and a 75 $\mathrm{km}$ fiber span. The circle set corresponds to $\mathrm{P}_{1}=\mathrm{P}_{2}=\mathrm{P}_{3}=50 \mathrm{~mW}$, the square set to $\mathrm{P}_{1}=\mathrm{P}_{2}=\mathrm{P}_{3}=100 \mathrm{~mW}$, and the triangle set to $\mathrm{P}_{1}=\mathrm{P}_{2}=\mathrm{P}_{3}=200 \mathrm{~mW}$.

In this scenario, there is a minimum power per pump, Pth, for which pump-pump interaction affects gain profile. The threshold dependence with co and counter- propagating configurations is shown in Fig. 4 and 5, respectively, where $\Delta G_{\text {on-off }}$ curves are shown for different pump power values for each configuration. For co-propagating configuration, $\Delta \mathrm{G}_{\mathrm{on-off}}$ is negative below around $1537 \mathrm{~nm}$ and positive above it, depicting a transfer of energy for power values as low as $20 \mathrm{~mW}$ per pump. The energy transfer contributes to around $1.5 \mathrm{~dB}$ gain difference for pump powers of $100 \mathrm{~mW}$. On the other hand, for the counter-propagating configuration, $\Delta \mathrm{G}_{\text {on-off }}$ negative to positive transition is observed only for power values above $60 \mathrm{~mW}$ per pump. Furthermore, the threshold power is independent of fiber length for co-propagating configuration and grows exponencially for the counterpropagating configuration, as shown in Fig.6. The counter-propagating configuration exponencial behavior is a consequence of the power being launched at the end of the fiber. The longer the fiber length the higher the power to surpass the losses, increasing threshold level. 
Journal of Microwaves, Optoelectronics and Electromagnetic Applications, Vol. 16, No. 3, September 2017832 DOI: http://dx.doi.org/10.1590/2179-10742017v16i31017

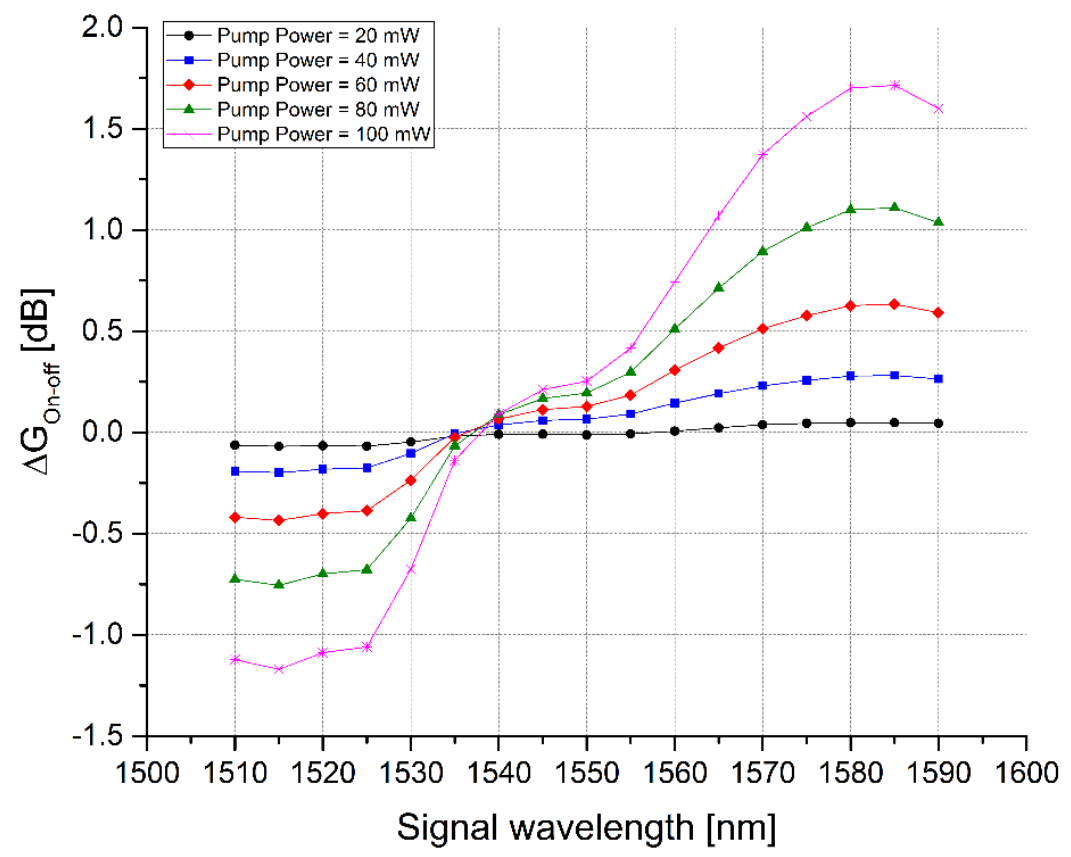

Fig.4 - On-Off gain difference for co-propagating pumps, $\Delta G_{\text {on-off }}$, for a $75 \mathrm{~km}$ fiber span. The power per pump in the curves are : circles $-20 \mathrm{~mW}$, squares $-40 \mathrm{~mW}$, diamond $-60 \mathrm{~mW}$, triangle $-80 \mathrm{~mW}$ and star $-100 \mathrm{~mW}$. P1=P2=P3 for each curve. 


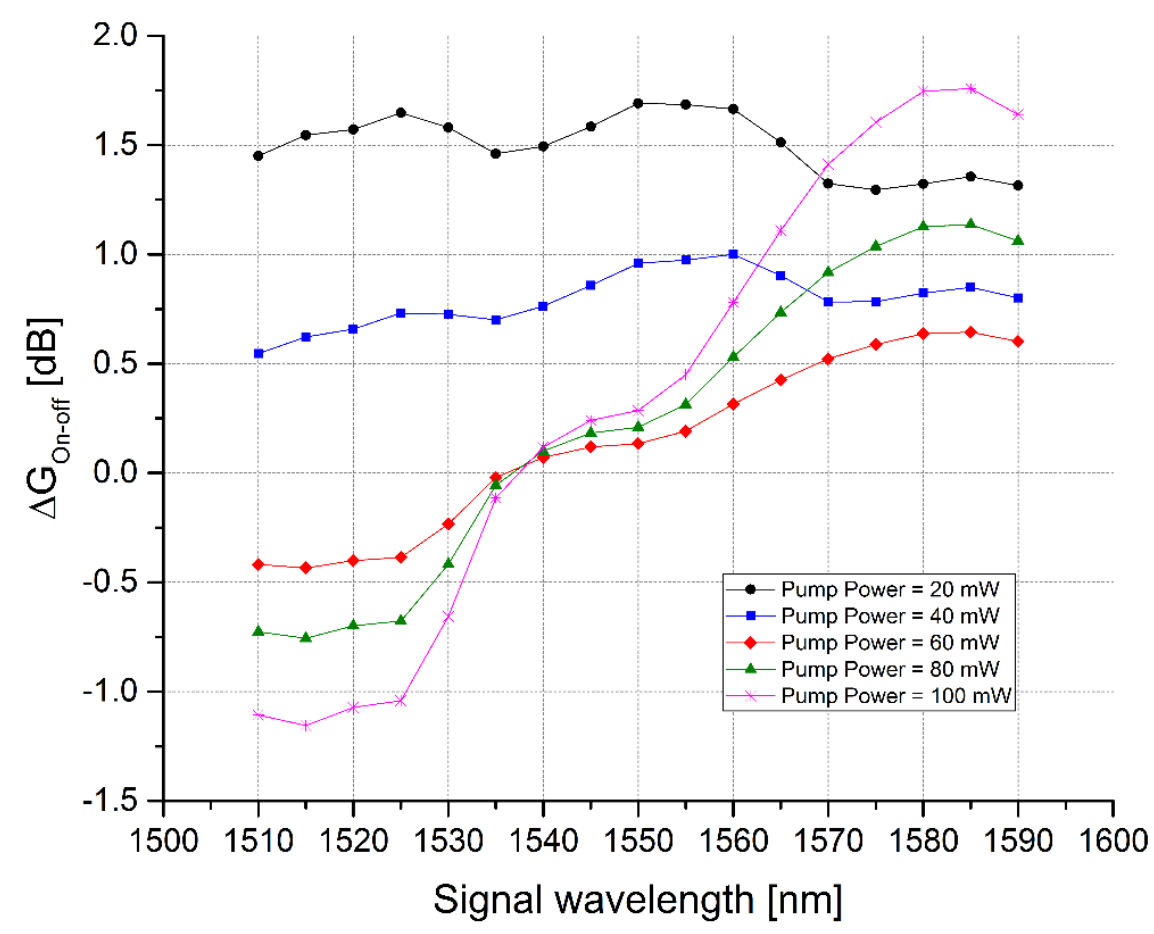

Fig.5 - On-Off gain difference for counter-propagating pumps, $\Delta G_{\text {on-off }}$, and a $75 \mathrm{~km}$ fiber span. The power per pump in the curves are : circles $-20 \mathrm{~mW}$, squares $-40 \mathrm{~mW}$, diamond $-60 \mathrm{~mW}$, triangle $-80 \mathrm{~mW}$ and star $-100 \mathrm{~mW} . \mathrm{P}_{1}=\mathrm{P}_{2}=\mathrm{P}_{3}$ for each curve.

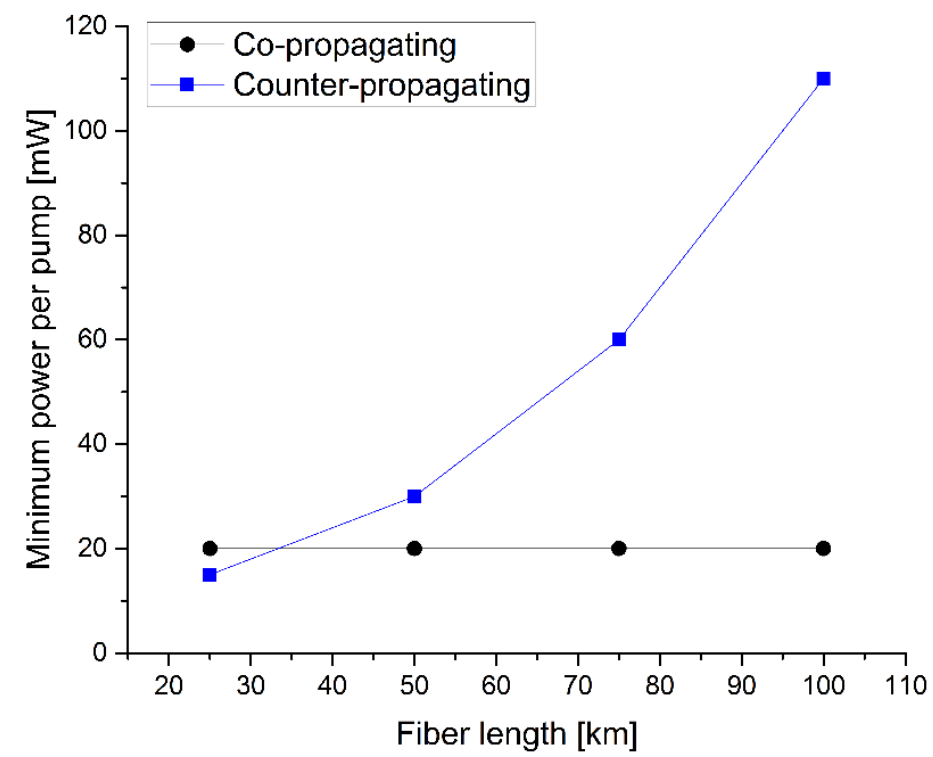

Fig.6 - Minimum power per pump, Pth, as a function of amplifier fiber length for co and counter- propagating configurations. 
In Fig. 7, the central pump power, P2, is reduced to half the value of P1 and P3, for the counterpropagating case discussed in Fig.5. A simple reduction by half leads to a decrease in pump power threshold, and consequently pump-pump interaction contribution to gain profile. Before the reduction the total threshold power was about $240 \mathrm{~mW}$ total $(80 \mathrm{~mW}$ per pump), and after $200 \mathrm{~mW}$ total $(80 \mathrm{~mW}$ for P1 and P3 and 40mW for P2).

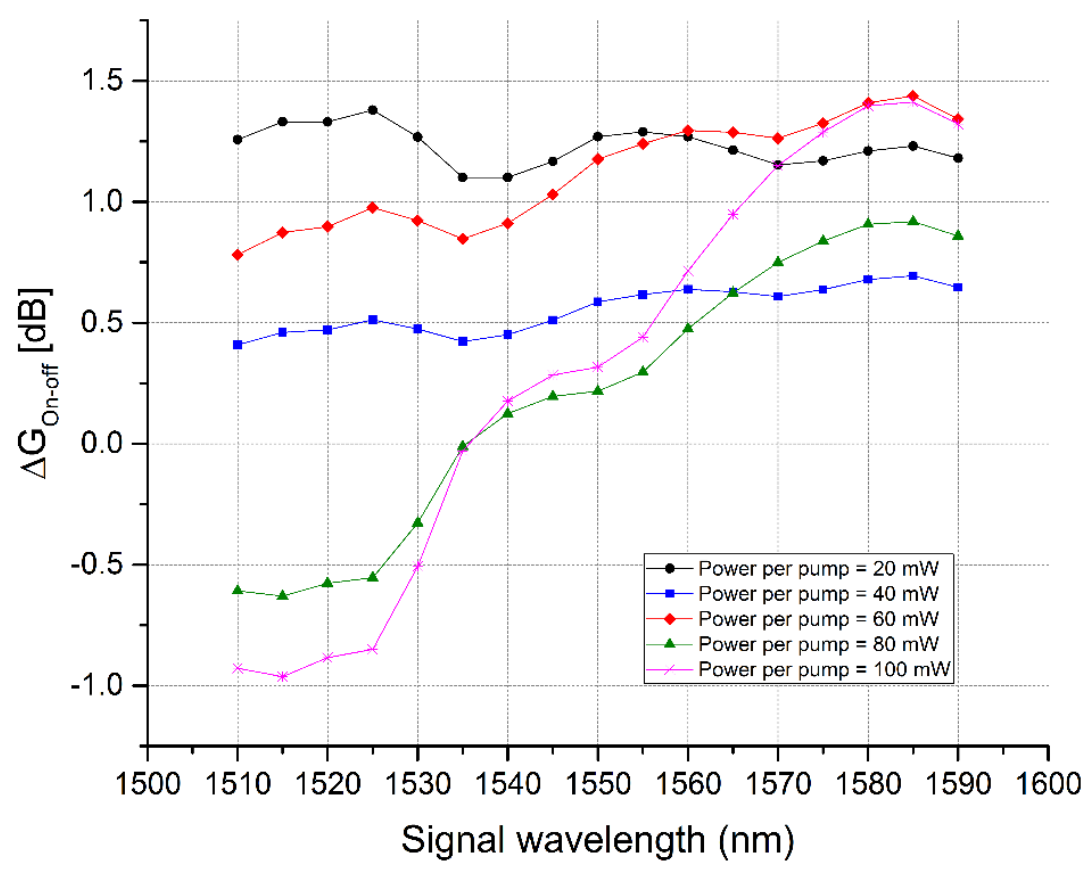

Fig. 7 - On-Off gain difference for counter-propagating pumps, $\Delta G_{o n-o f f}$, and a $75 \mathrm{~km}$ fiber span. The power per pump in the curves are : circles $-20 \mathrm{~mW}$, squares $-40 \mathrm{~mW}$, diamond $-60 \mathrm{~mW}$, triangle $-80 \mathrm{~mW}$ and star $-100 \mathrm{~mW} . \mathrm{P}_{1}=\mathrm{P}_{3}$ and $\mathrm{P}_{2}=$ $(1 / 2) \mathrm{P}_{3}$ for each curve.

The correlation between gain ripple and $\max \left|\Delta G_{\text {On-Off }}\right|$ for the scenario described in Fig. 7 is better viewed by the corresponding relative difference parameter, $\Delta \%$, as a function of $\mathrm{P}_{2} / \mathrm{P}_{3}$ power ratio as presented, in Fig.8. The minimum $\Delta \%$ occurs for a $\mathrm{P}_{2} / \mathrm{P}_{3}$ on the order of 0.6 for $\mathrm{P}_{1}$ and $\mathrm{P}_{3}$ values ranging from $60 \mathrm{~mW}$ up to $100 \mathrm{~mW}$ and 0.7 for $\mathrm{P}_{1}$ and $\mathrm{P}_{3}$ on the order of $40 \mathrm{~mW}$. For a $75 \mathrm{~km}$ fiber length $\Delta \%$ at minimum is on the order of $10 \%$. Nonetheless, it decreases with fiber length reaching about $1 \%$ for $\mathrm{L}=25 \mathrm{~km}[17]$.

The relative difference, $\Delta \%$, dependency with fiber length for $\mathrm{P}_{2} / \mathrm{P}_{3}=0.4,0.6$ and 0.8 for $\mathrm{P}_{1}$ and $\mathrm{P}_{3}$ equal to $100 \mathrm{~mW}$ is shown in Fig 9. It is practically independent of fiber length for $\mathrm{P}_{2} / \mathrm{P}_{3}=0.4$ and 
$\mathrm{P}_{2} / \mathrm{P}_{3}=0.8$, and on the order of $19 \%$ and $27 \%$ respectively. Otherwise, for $\mathrm{P}_{2} / \mathrm{P}_{3}=0.6$, it shows a considerable dependency on fiber length, decreasing as total length varies from $20 \mathrm{~km}$ up to $45 \mathrm{~km}$. A minimum relative difference of $1 \%$ is reached at $45 \mathrm{~km}$ fiber length. Then, it increases for lengths up to $85 \mathrm{~km}$. Therefore, there is an optimum $\mathrm{P}_{2} / \mathrm{P}_{3}$ ratio and fiber length for which the values of ripple and $\max \left|\Delta G_{\text {on-off }}\right|$ have an agreement of about $1 \%$. In summary, Fig. 9 shows a strong relation between ripple and pump-pump interaction for fiber lengths from $30 \mathrm{~km}$ up to $80 \mathrm{~km}$ (smaller than $5 \%$ ) when the central pump power is reduced by $40 \%$ with respect to the value of $\mathrm{P}_{1}$ and $\mathrm{P}_{3}$.

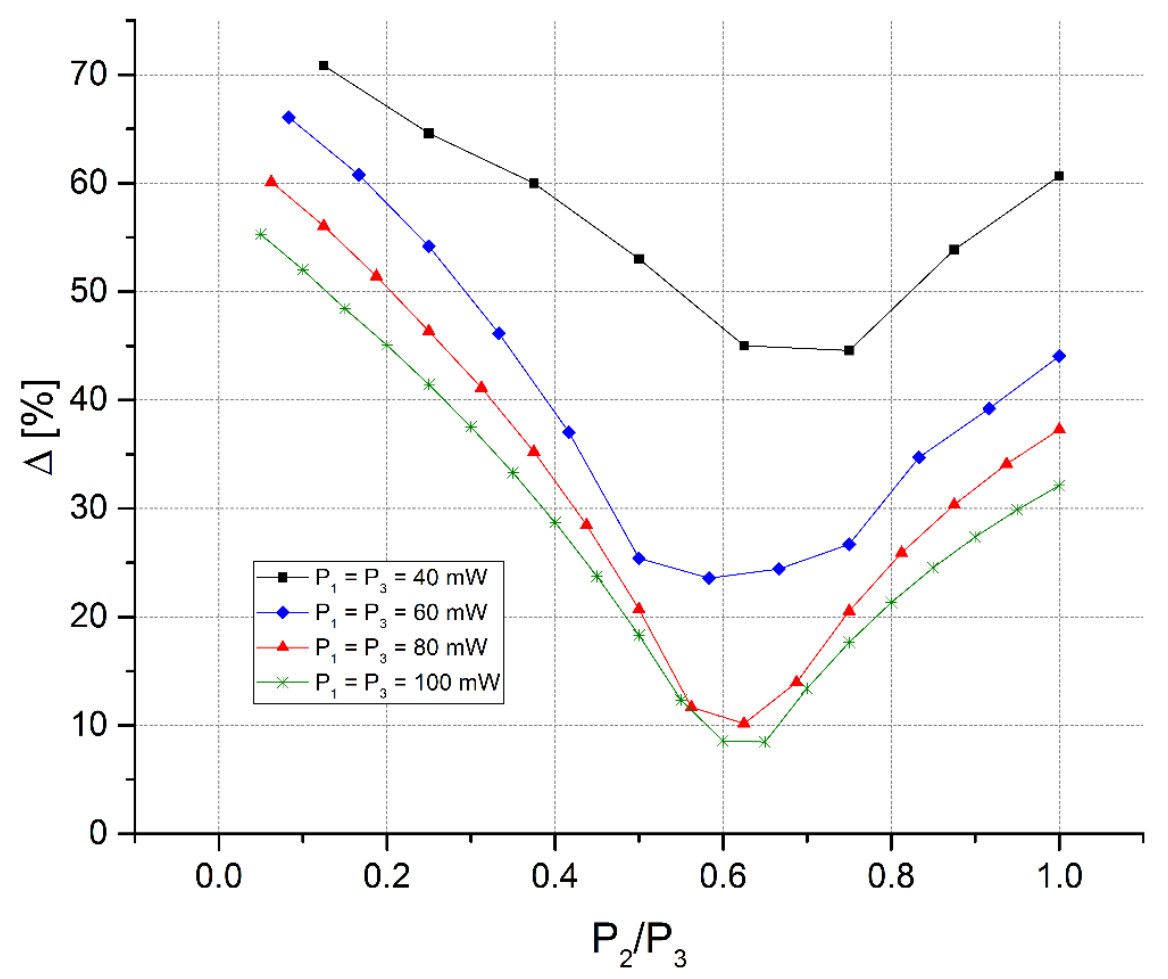

Fig.8 $-\Delta \%$ as a function of $\mathrm{P}_{2} / \mathrm{P}_{3}$ power ratio and a $75 \mathrm{~km}$ fiber span. The power per pump $\mathrm{P}_{1}$ and $\mathrm{P}_{3}$ in the curves are : squares $-40 \mathrm{~mW}$, diamond $-60 \mathrm{~mW}$, triangle $-80 \mathrm{~mW}$ and star $-100 \mathrm{~mW}$. 
Journal of Microwaves, Optoelectronics and Electromagnetic Applications, Vol. 16, No. 3, September 2017836 DOI: http://dx.doi.org/10.1590/2179-10742017v16i31017

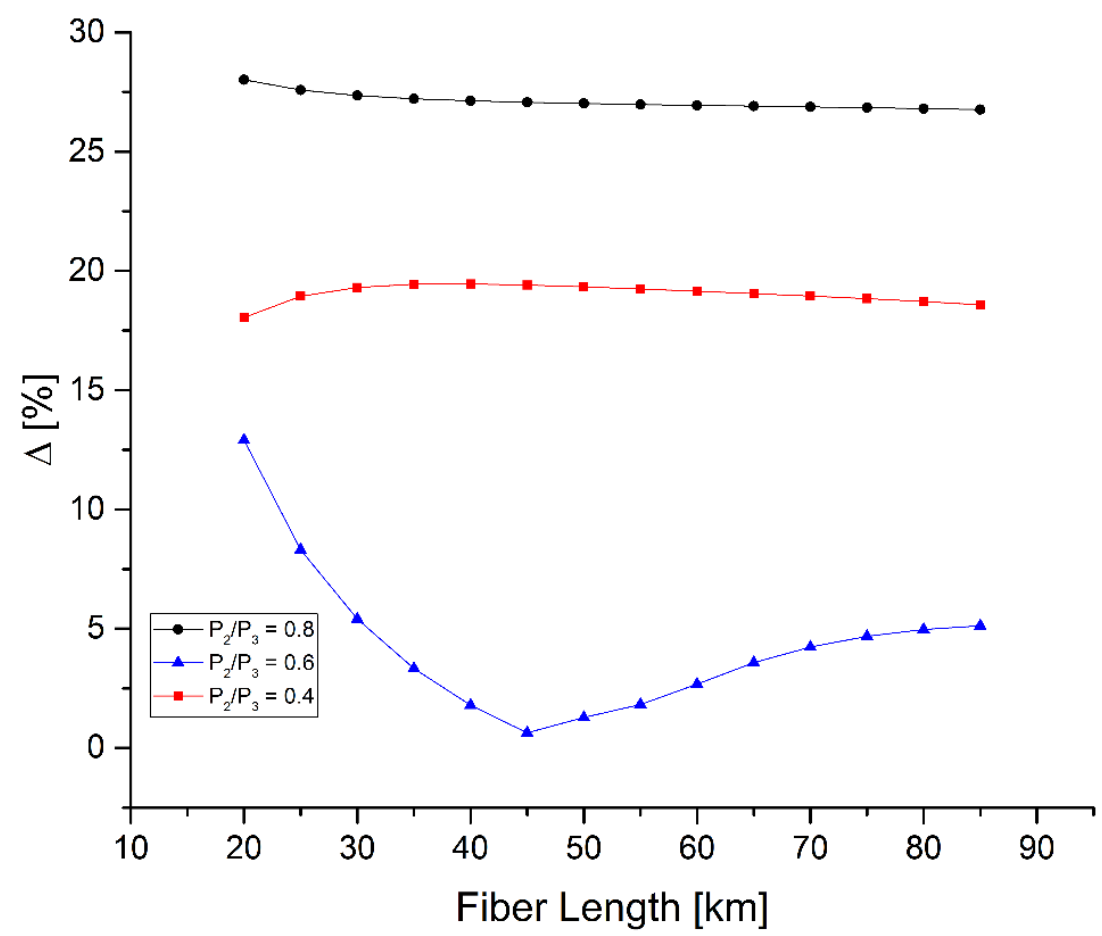

Fig.9 $-\Delta \%$ as a function of fiber span length. The $\mathrm{P}_{2} / \mathrm{P}_{3}$ power ratio in the curves are: 0.4 (square), 0.6 (triangle) and 0.8 (circle). P1 and P3 are set equal to $100 \mathrm{~mW}$. Counter-propagating configuration.

In Fig. 10 it is shown simultaneously the on-off gain and gain ripple as a function of $\mathrm{P}_{2} / \mathrm{P}_{3}$ ratio, for values of $\mathrm{P}_{1}$ and $\mathrm{P}_{3}$ equal to 60,80 and $100 \mathrm{~mW}$ for $75 \mathrm{~km}$. The highlighted area depicts the below $1 \mathrm{~dB}$ ripple area, with minimum ripple of $0.67 \mathrm{~dB}$ for a $3.77 \mathrm{~dB}$ gain, and a total pump power of $150 \mathrm{~mW}$ for the $75 \mathrm{~km}$ fiber span. 


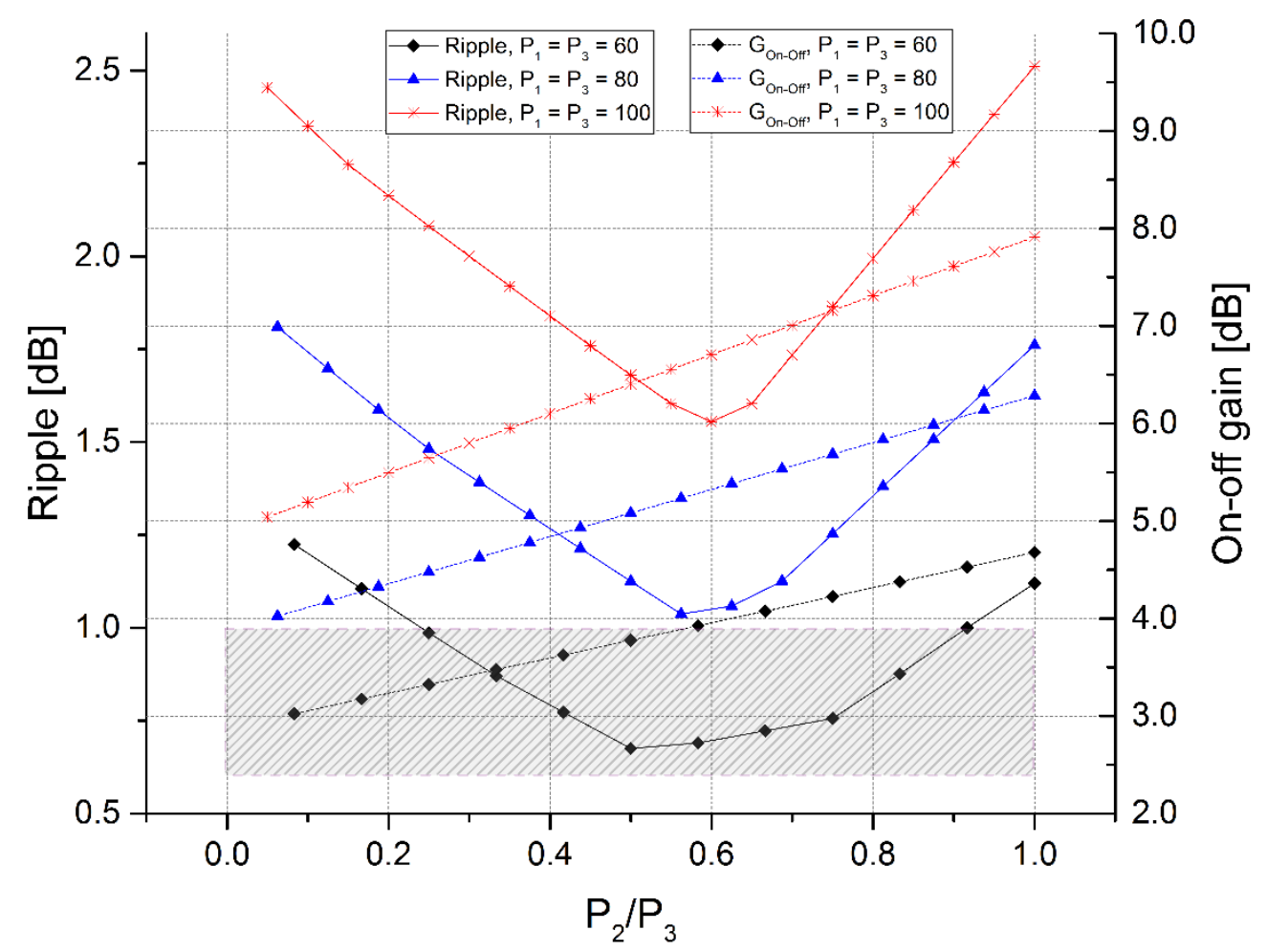

Fig.10 - Gain ripple (left axis and solid curves) and on-off gain (right axis and dashed curves) as a function of $\mathrm{P}_{2} / \mathrm{P}_{3}$ power ratio and a $75 \mathrm{~km}$ fiber span. The power per pump $\mathrm{P}_{1}$ and $\mathrm{P}_{3}$ in the curves are: $60 \mathrm{~mW}$ (diamond), $80 \mathrm{~mW}$ (triangle), and $100 \mathrm{~mW}$ (star).

\section{CONCLUSIONS}

A novel strategy was introduced in order to simplify the design of a broadband, medium level power and low power consumption (300mW maximum) Raman amplifier. It isolates pump-pump interaction contribution to gain profile, and its relation to the amplifier gain ripple, allowing for ripple minimization by tailoring pump-pump interaction through a single design parameter, the relative central pump power level. When the maximum value of the on-off gain difference is compared with the ripple values, the results also show that the relative difference between them is lower (e. g.: they have a better agreement) for central pump power values between 40 and $70 \%$ of the external pumps. The approach was applied to a low water peak fiber, in co and counter-propagation configurations and fiber lengths up to $75 \mathrm{~km}$. A reduction in central pump power around $40 \%$ relative to the external ones reduces pump-pump interaction and consequently the gain ripple as well. The results demonstrated a $4 \mathrm{~dB}$ gain over an $80 \mathrm{~nm}$ band, with a ripple better than $0.70 \mathrm{~dB}$. Finally, the approach described here, although simplistic for a full design of practical amplifiers, could be used as initial step of a complex robust optimization routine to limit the solution space, and possibly achieve a faster conversion method. 


\section{APPENDIX}

The Raman nonlinear equation used to simulate the amplifier performance takes into account the Raman effect as well as signal-signal interaction, pump-pump interaction, Double Rayleigh Backscattering, and Amplified Spontaneous Emission (ASE). The following equations are built-in into the code:

$$
\begin{aligned}
& \frac{d P_{s, i}}{d z}=\sum_{\lambda i>\lambda j} C_{i, j}^{R}\left(P_{p, j}^{f}+P_{p, j}^{b}\right) P_{s, i}+2 \sum_{\lambda i>\lambda j} C_{i, j}^{R} N_{i}\left(P_{p, j}^{f}+P_{p, j}^{b}\right)+\sum_{\lambda k<\lambda i} C_{k, i}^{R} P_{s, k} P_{s, i}- \\
& -\sum_{\lambda k>\lambda i} \frac{v_{s, k}}{v_{s, i}} C_{k, i}^{R} P_{s, k} P_{s, i}-\alpha_{R} S . P_{B S, i}-\alpha_{s, i} \cdot P_{s, i} \\
& \frac{d P_{p, j}^{f}}{d z}=-\sum_{\lambda i>\lambda j} \frac{v_{p, j}}{v_{s, i}} C_{i, j}^{R} \cdot P_{p, j}^{f} \cdot P_{s, i}-4 \sum_{\lambda i>\lambda j} \frac{v_{p, j}}{v_{s, i}} C_{i, j}^{R} N_{i} \cdot P_{p, j}^{f}+\varepsilon \sum_{\lambda k<\lambda j} C_{j, k}^{R}\left(P_{p, k}^{b}+P_{p, k}^{f}\right) \cdot P_{p, j}^{f}- \\
& -\varepsilon \sum_{\lambda k>\lambda j} \frac{v_{p, k}}{v_{p, j}} C_{k, j}^{R}\left(P_{p, k}^{b}+P_{p, k}^{f}\right) \cdot P_{p, j}^{f}-\sum_{\lambda k>\lambda j} \frac{v_{p, j}}{v_{s, i}} C_{k, j}^{R}\left(P_{B S, i}+P_{D B S, i}\right) \cdot P_{p, j}^{f}-\alpha_{p, j} \cdot P_{p, j}^{f} \\
& \frac{d P_{p, j}^{b}}{d z}=+\sum_{\lambda i>\lambda j} \frac{v_{p, j}}{v_{s, i}} C_{i, j}^{R} \cdot P_{p, j}^{b} \cdot P_{s, i}+4 \sum_{\lambda i>\lambda j} \frac{v_{p, j}}{v_{s, i}} C_{i, j}^{R} N_{i} \cdot P_{p, j}^{b}-\varepsilon \sum_{\lambda k<\lambda j} C_{j, k}^{R}\left(P_{p, k}^{b}+P_{p, k}^{f}\right) . P_{p, j}^{b}+ \\
& +\varepsilon \sum_{\lambda k>\lambda j} \frac{v_{p, k}}{v_{p, j}} C_{k, j}^{R}\left(P_{p, k}^{b}+P_{p, k}^{f}\right) \cdot P_{p, j}^{b}+\sum_{\lambda k>\lambda j} \frac{v_{p, j}}{v_{s, i}} C_{k, j}^{R}\left(P_{B S, i}+P_{D B S, i}\right) \cdot P_{p, j}^{b}+\alpha_{p, j} \cdot P_{p, j}^{b} \\
& \frac{d P_{B S, i}}{d z}=-\sum_{\lambda i>\lambda j} C_{i, j}^{R}\left(P_{p, j}^{f}+P_{p, j}^{b}\right) P_{B S, i}-4 \sum_{\lambda i>\lambda j} C_{i, j}^{R} N_{i}\left(P_{p, j}^{f}+P_{p, j}^{b}\right)-\sum_{\lambda k<\lambda i} C_{k, i}^{R}\left(P_{B S, k}+P_{D B S, k}\right) \cdot P_{B S, i}+ \\
& +\sum_{\lambda k>\lambda i} \frac{v_{s, k}}{v_{s, i}} C_{k, i}^{R}\left(P_{B S, k}+P_{D B S, k}\right) \cdot P_{B S, i}-\alpha_{R} S . P_{s, i}+\alpha_{R} S . P_{D B S, i}+\alpha_{s, i} \cdot P_{B S, i} \\
& \frac{d P_{D B S, i}}{d z}=+\sum_{\lambda i>\lambda j} C_{i, j}^{R}\left(P_{p, j}^{f}+P_{p, j}^{b}\right) P_{D B S, i}+4 \sum_{\lambda i>\lambda j} C_{i, j}^{R} N_{i}\left(P_{p, j}^{f}+P_{p, j}^{b}\right)+\sum_{\lambda k<\lambda i} C_{k, i}^{R}\left(P_{B S, k}+P_{D B S, k}\right) \cdot P_{D B S, i}- \\
& -\sum_{\lambda k>\lambda i} \frac{v_{s, k}}{v_{s, i}} C_{k, i}^{R}\left(P_{B S, k}+P_{D B S, k}\right) \cdot P_{D B S, i}+\alpha_{R} S \cdot P_{B S, i}-\alpha_{s, i} \cdot P_{D B S, i}
\end{aligned}
$$

In equations (2) to (6), $P_{s, i}$ is the signal power at wavelength $\lambda_{i}, P_{p, j}^{f}$ is the forward pumping power at wavelength $\lambda_{j}, P_{p, j}^{b}$ is the backward pumping power at wavelength $\lambda_{j}, P_{B S, i}$ is the Rayleigh scattered signal power at wavelength $\lambda_{i}, P_{D B S, i}$ is the Double Rayleigh scattered signal power at wavelength $\lambda_{i}, C_{i, j}^{R}$ is the Raman gain coefficient of a pump at wavelength $\lambda_{j}$ in a signal at wavelength $\lambda_{i}, \alpha_{R}$ is the Rayleigh Scattering coefficient, $S$ is the Rayleigh Backscattering capture factor, $\alpha_{s, i}$ is 
the fiber attenuation coefficient for a signal at wavelength $\lambda_{i}, \alpha_{p, i}$ is the fiber attenuation coefficient for a pump at wavelength $\lambda_{j}, N_{i}$ is the noise power at wavelength $\lambda_{i}, v_{s, i}$ is the frequency of the signal at wavelength $\lambda_{i}, v_{p, k}$ is the frequency of the pump at wavelength $\lambda_{k}$ and $\epsilon$ is a parameter which value is either equal to 1 ( pump-pump interaction effects area accounted in the simulation) or 0 (pump-pump interaction effects are not accounted in the simulation).

\section{ACKNOWLEDGMENT}

The authors wish to acknowledge the Coordenação de Aperfeiçoamento de Pessoal de Nível Superior (CAPES), Conselho Nacional do Desenvolvimento Científico e Tecnológico (CNPq) and Fundação Carlos Chagas Filho de Amparo à Pesquisa do Estado do Rio de Janeiro (FAPERJ) for partially supporting this work.

\section{REFERENCES}

[1] A. Jirattigalachote, "Provisioning Strategies for Transparent Optical networks Considering Transmission Quality, Security, and Energy Efficiency”, PhD Thesis in Microelectronics and Applied Physics, Royal Institute of Technology (KTH), Sweden, 2012.

[2] J. D. Añia-Castañon et al, "Simple design method for gain-flattened three- pump Raman amplifiers”. Optical and Quantum Electronics, 39, issue 3, pp. 213-220 .Springer, 2007.

[3] J. Hu, B. S. Marks, C. R. Menyuk; "Flat-Gain Fiber Raman Amplifiers Using Equally Spaced Pumps". Journal of Lightwave Technology, 22(6), pp. 1519-1522, 2004.

[4] S. M. Bilal, M. Zafrullah, M. K. Islam., "Gain Flattening of DWDM channels for the entire C \& L bands”. Journal of Optical Technology, 75, n. 9, pp. 557-560, September 2012.

[5] B. Lee, X. Liu, "Optimal Design for Ultra-Broad-Band Amplifier". Journal of Lightwave Technology, 21, n. 12, pp 3446-3455, December 2003.

[6] X. Liu et al, "Optimizing gain profile and noise performance for distributed fiber Raman amplifiers". Optics Express, 12, n. 24, pp. 6053-6066, November 2004.

[7] V. E. Perlin and H. G. Winful, "Optimal Design of Flat-Gain Wide-Band Fiber Raman Amplifiers". Journal of Lightwave Technology, 20, n. 2, pp. 250-254, February 2002.

[8] Z. LI et al, "Raman amplifier design using geometry compensation technique". Optics Express, 12, no 3, pp. 436-441, February 2004.

[9] G. C. M. Ferreira et al, "Optimization of Distributed Raman Amplifiers Using a Hybrid Genetic Algorithm With Geometric Compensation Technique”. IEEE Photonics Journal 3, n. 3, pp. 390-399, June 2011. 
[10] C. J. A. Bastos-Filho, "Simple Design of Raman Fiber Amplifiers using a Multi-objective Optimizer". 11th International Conference on Intelligent Systems Design and Applications, pp. 1128-1133, November 2011.

[11] F. Emami, M. Akhlaghi, "Gain Ripple Decrement of S-Band Raman Amplifiers". IEEE Phot. Tech. Letter, 24 n. 15, pp. 1349-1352, August 2012.

[12] F. M. Mustafa, A. A. M. Khalaf, F. A. El-Geldawy, "Multi-pumped Raman Amplifier for Long-Haul UW-WDM Optical Communication Systems: Gain Flatness and Bandwidth Enhancements". 15th International Conference on Advanced Computing Technologies (ICACT), pp. 122-127, 2013.

[13] H. Kidorf, K. Rottwitt, M. Nissov et al, "Pump interactions in a 100-nm bandwidth Raman amplifier", IEEE Phot. Tech. Letter, 11, pp. 530- 532, May 1999.

[14] L. A. M. Saito et al, "Multi-pump discrete Raman amplifier for CWDM system in the Oband", Optical Fiber Technology, 14, pp. 294-298, October 2008.

[15] Stolen and E. P. Ippen, "Raman gain in glass optical waveguides". Appl. Phys. Lett., 22, pp. 276-279, 1973.

[16] R. F. R. de Farias et al, "Study of the quantitative impact of pump-pump interaction in wideband Raman amplifiers in the S, C and L bands". Proceedings of SPIE, 7721. April 2010.

[17] R. S. Santos et al, "On the Relation Between Gain Ripple and Pump-Pump Interaction in the Design of Broad Band Raman Amplifiers". 16 SBMO - Simpósio Brasileiro de Micro-ondas e Optoeletrônica e $11^{\circ}$ CBMag - Congresso Brasileiro de Eletromagnetismo (MOMAG 2014), pp. 643-646, 2014. 